\title{
P 128 COMPARING THE ACCURACY OF AN ONLINE PROGNOSTICATION TOOL WITH CLINICAL TEAM PROGNOSTICATION
}

Sarah Forrest, ${ }^{1,2}$ Ann Holmes, ${ }^{1}$ Eva Lew ${ }^{1} .{ }^{1}$ Farleigh Hospice, Chelmsford, UK; ${ }^{2} G P$ Unit, Institute for Public Health, School of Clinical Medicine, University of Cambridge

10.1136/bmjspcare-2014-000654.169

Background Medical Teams regularly formulate a prognosis for patients to allow appropriate plans to be made and to help patients and families prepare for end of life.

Research shows that clinicians are over-optimistic in prognosis formulation to a degree of up to 5 times the actual time to death. ${ }^{1}$

The 'Prognosis in Palliative Care Study' (PiPS) online prognostication tool allows clinicians to input data and receive a prognosis in days, weeks or months, and has been found to be at least as accurate when compared with physician estimate alone. $^{2} 3$

Aim To compare the PiPS-A online prognostication tool with Clinician estimate of prognosis and the actual time to death for all advanced cancer patients admitted to the IPU over three months.

To assess whether using PiPS-A improves the accuracy of prognostication in our patient group compared with the team's estimate, and whether using it adds to our practice.

Results 33 PiPs-A forms were completed on 32 patients over 3 months from April-July 2012.

Clinician prognostications were at least as accurate as PiPs-A supported prognostication. For patients who had an actual prognosis of weeks or months, the clinician (54\%/55\%)was markedly more accurate than the PiPs-A (30\%/31\%). PiPs-A was as good as a clinician in predicting days ( $80 \%$ vs $78 \%)$.

Discussion Doctors find prognostication difficult, yet it is central to assessment and management of patient and in planning for changing care needs. PiPs-A may be useful in prognostication of patients in whom prognosis may be short and the efficacy of interventional treatments is questioned.

Junior doctors have little experience of formulating an accurate prognosis; PiPS has a role as a teaching aid for juniors to enable them to develop this skill.

\section{REFERENCES}

1 Extent and determinants of error in doctors' prognoses in terminally ill patients. Christakis \& Lamont: BMJ 2000;320:469-73.

2 Development of Prognosis in Palliative Care Study (PiPS) predictor models to improve prognostication in Advanced Cancer: prospective cohort study. Gwilliam et al: BMJ Supportive and Palliative Care 2012;2:63-71.

3 www.pips.sgul.ac.uk 